\title{
Prognostic parameters of luminal A and luminal $B$ intrinsic breast cancer subtypes of Pakistani patients
}

\author{
Atif Ali Hashmi', Saher Aijaz², Saadia Mehmood Khan', Raeesa Mahboob', Muhammad Irfan', Narisa Iftikhar Zafar ${ }^{1}$, \\ Mariam Nisar ${ }^{1}$, Maham Siddiqui ${ }^{3}$, Muhammad Muzzammil Edhi ${ }^{5}$, Naveen Faridi ${ }^{4}$ and Amir Khan ${ }^{6^{*}}$ (D)
}

\begin{abstract}
Background: Prognosis of breast cancer and success of therapeutic interventions largely rely on the clinico-pathologic and biological characteristics of the tumor and vary due to the heterogeneous nature of breast cancers. The aim of this study was to determine the frequency and prognostic parameters of luminal breast cancers in our population to devise targeted and personalized therapeutic regimens tailored to the needs of the loco-regional population.

Methods: A retrospective cross-sectional study including 1951 cases of primary breast cancer treated at Liaquat National Hospital Karachi was conducted during the year 2011-2016. The clinico-pathologic characteristics were observed and semiquantitative immunohistochemical analysis was performed to study the luminal subtypes A and B. The cross-tabulated statistics of the observed characteristics were performed between the two subtypes. The significance level of each characteristic was estimated utilizing the chi-square test.

Results: Luminal cancers comprised $62.7 \%$ of the total number of cases diagnosed with breast cancers in the study period. Out of these 1224 cases of luminal cancers, 845 cases (69\%) were luminal B, while 379 (31\%) cases were of luminal A. Luminal B cancers were significantly more common in younger age groups as compared to luminal A cancers. Comparison of the two subtypes of luminal breast cancers revealed significant differences. Luminal B cancers were associated with higher grade (26\% grade III in luminal B compared to $8 \%$ in luminal A), micropapillary histology, and high frequency of nodal metastasis (54 vs. 43\%).

Conclusions: Luminal B comprised the most frequent subtype of breast cancer in our study and they were found more constantly in a younger age group. Moreover, they were associated with adverse clinico-histologic parameters like higher grade and nodal metastasis. Therefore, we suggest that, despite lack of widespread availability of molecular studies in our setup, IHC-based typing should be done in every case of breast cancer to individualize therapy.
\end{abstract}

Keywords: Luminal A, Luminal B, ER, PR, Her2neu, Breast cancer

\section{Background}

Luminal breast cancers were first defined by molecular profiling as tumors with gene expression similar to the luminal epithelium of the breast. Luminal A tumors are marked by high expression of estrogen receptor(ER)-related genes, low expression of human epidermal growth factor receptor 2 (HER2neu) genes and low expression of proliferation-related genes $[1,2]$. On the other hand, luminal B tumors exhibit low expression of ER-related

\footnotetext{
* Correspondence: dramirkhan04@gmail.com

${ }^{6}$ Department of Medicine, Kandahar University, Kandahar, Afghanistan Full list of author information is available at the end of the article
}

genes, variable expression of HER2neu-related genes, and high expression of proliferation-related genes with worse prognosis than luminal A tumors [3]. St. Gallen International Expert Consensus on the "Primary Therapy of Early Breast Cancer 2013" proposed that luminal subtypes of breast cancer can be defined in view of immunohistochemistry (IHC) for ER, progestrone receptor (PR), HER2neu, and ki67 index rather than conventional molecular diagnostics [4]. Ki67 index reflects the proportion of the proliferation genes/cells and thus employed to subtype luminal tumors. Therefore, Ki67 index is also utilized to tailor the therapy for the patient as in some cases 
chemotherapy or endocrine therapy alone is inadequate [5]. Although exact cutoffs for ki67 and PR were not defined in St. Gallen International Expert Consensus, however, some proposals were given, stating that a cutoff value of $<14 \%$ ki67 can be used to define luminal cancers in the absence of HER2neu expression based on single reference laboratory [6]. On the other hand, Prat et al. expressed that a cutoff value of $>20 \%$ can be adapted to ascertain luminal A cancers. These proposals were derived from the results of five cohorts, which were combined and interpreted by experts [7]. It is also well established that enormous heterogeneity exists in genetic and morphological characteristics of breast tumors. On account of this heterogeneity, therapeutic regimens must be tailored to the loco-regional breast cancer profiles. Studies have demonstrated a different behavior of breast cancer in Pakistan with higher prevalence in a younger age group with aggressive nature and poor survival rates [8-10]. Therefore, we aimed to determine prognostic parameters of luminal breast cancers in our population which can help in devising targeted and personalized therapeutic regimens tailored to the needs of the loco-regional population.

\section{Methods}

It was a retrospective cross-sectional study including 1951 cases of primary breast cancer treated at Liaquat National Hospital Karachi from the year 2011 till 2016. An approval from an ethical review committee was taken antecedent to conducting the study. Specimens included trucut biopsies, wide local excisions + lymph node dissection, or simple/modified radical mastectomies based on sentinel lymph node status. Histopathologic characteristics recorded include tumor type, size, grade, and lymph node status along with degree of necrosis, lymphocytic reaction, and fibrosis. Lymphocytic infiltration (tumor infiltrating lymphocytes) was assessed in the stromal compartment of the tumor. Percentage of stromal area showing dense mononuclear infiltrate was evaluated and scored as a continuous variable. However, as no clinically relevant threshold was defined yet, therefore, we took cutoffs of $<10 \%$ as mild to moderate and $>50 \%$ as severe lymphocytic infiltration [11]. Lymphovascular invasion was assessed outside the borders of invasive carcinoma. The tumor emboli should not exactly conform to the contours of the space in which they were found. In addition, endothelial cell nuclei should also be identified [12]. One representative section is selected for IHC studies including ER, PR, her2neu, and ki67 by DAKO envision method.

The results for ER and PR were scored in a semiquantitative fashion incorporating both the intensity and the distribution of specific staining [13]. For each tissue, intensity and percentage of positive staining cells were determined. In addition, a value designated as H-SCORE was derived by summing up the percentage of cell staining multiplied by the weighted intensity of staining (negative-0, weak-1, moderate-2, strong-3) with 300 possible values (1-300) [14]. More than 1\% staining cells was considered positive expression.

HER2neu were scored based on the intensity and percentage of positive cells on a scale of 0 to $3+$ according to American Society of Clinical Oncology/College of American Pathologists (ASCO/CAP) guidelines. Cases were reported 0 (negative) if no staining observed or incomplete, faint/barely perceptible membrane staining in $\leq 10 \%$ of invasive tumor cells; $1+$ (negative) if incomplete, faint/barely perceptible membrane staining in > $10 \%$ of invasive tumor cells; $2+$ (positive) if incomplete and/or weak to moderate circumferential membrane staining in $>10 \%$ of invasive tumor cells or complete, intense, circumferential membrane staining in $\leq 10 \%$ of invasive tumor cells; or $3+$ (positive) if complete, intense, circumferential membrane staining in $>10 \%$ of invasive tumor cells $[15,16]$. Cases with intermediate $(2+)$ expression of Her2neu underwent subsequent fluorescence in situ hybridization (FISH) testing for Her2neu gene amplification. FISH testing was performed using FDA-approved Path Vysion Her2 DNA Probe kit.

Results were expressed as the ratio of Her2 signals as compared to CEP 17 signals according to ASCO/CAP guidelines. A result of HER2/CEP17 ratio $<2.0$ and average HER2 copy number $<4.0$ signals/cell is considered negative (not amplified); HER2/CEP17 ratio $<2.0$ and average HER2 copy number $\geq 4.0$ but $<6.0$ signals/cell as equivocal; and HER2/CEP17 ratio $\geq 2.0$ (regardless of average HER2 copy number) or average HER2 copy number $\geq 6.0$ signals/cell (regardless of ratio) as positive (amplified).

Ki-67 immunoreactivity was recorded as continuous variables, based on the proportion of positive tumor cells (0-100\%). At least 1000 cells were evaluated before calculating an average estimate. If hot spots were present, then they are included in the estimation. Ki67 index was further categorized into four groups, < 14, 15-24, 25-44, and $>45 \%$.

Luminal A and luminal B breast cancers were defined as follows:

Luminal A: ER and/or PR positive, ki67 low (<14\%).

Luminal B: ER and/or PR positive with ki67 high (> $14 \%$ ) or ER and/or PR positive and Her2neu positive irrespective of ki67.

All cases of primary breast cancers which were classified as luminal according to the above criteria were included in the study. Cases of recurrent breast cancers and those with prior chemotherapy were excluded from the study.

Statistical package for social sciences (SPSS 21) was used for data compilation and analysis. Mean and 
standard deviation were calculated for quantitative variables. Frequency and percentage were calculated of qualitative variables. Shapiro-Wilk test was applied to test normality. Chi-square was applied to see association. Mann-Whitney test was applied to compare difference in means among groups by considering $P$ value $\leq 0.05$ as significant.

\section{Results}

A total of 1951 cases of primary breast cancer diagnosed at our facility were assessed for inclusion and exclusion criteria. One thousand two hundred twentyfour cases were included in our study as they met the diagnostic criteria of luminal type cancers which were estrogen receptor and progesterone receptor positive. Luminal cancers comprised $62.7 \%$ of the total number of cases diagnosed with breast cancers. Out of these 1224 cases, 845 cases, i.e., $69 \%$ of the samples, were luminal $\mathrm{B}$, while 379 , i.e., $31 \%$ of the cases, were luminal A. Table 1 shows the comparison of these two categories of luminal cancers.

Mean age for luminal B cancers was found to be 51.1 years, which was significantly lower in relation with luminal A cancers for which the mean age was 54.6 years. Notably, luminal B cancers were of high grade as compared to luminal A cancers as the frequency of grade 3 tumors in luminal B was found to be $26.5 \%$, while in luminal $\mathrm{B}$, the frequency was only $8.4 \%$. Similarly, nodal metastasis was more prominent in luminal B as $54 \%$ of luminal B cases had nodal metastasis and only $43 \%$ of luminal A had nodal metastasis $(P$ value $<0.05)$.

As far as biomarker status is concerned, $73 \%$ of luminal cancers were PR positive as compared to $83 \%$ positivity in luminal A cancers. Table 2 shows comparison of histologic features of luminal A and B cancers. Lymphovascular invasion was seen more frequently in luminal B cancers compared to luminal A cancers.

The histologic subtyping of the luminal A and luminal B cancers also showed significant variations. Micropapillary histology was noted more frequently in luminal B subtype while frequency of lobular, cribriform, papillary, and mucinous tumors was higher in luminal A subtype of breast cancers. Table 3 depicts the distribution of different histologic subtypes of breast cancer in these two categories of luminal cancers.

\section{Discussion}

In Asian and African regions, the incidence of breast cancer among women below the age of 40 years is $30 \%$, which is significantly high as compared to the rest of the world [17-19]. Therefore, the present study investigated the prognostic parameters of luminal breast cancers in Pakistani population, to assist the physicians in tailoring targeted and personalized hormonal and chemotherapeutic interventions. Acknowledged by previous studies, gene expression profiling helps in identifying biologically distinct breast cancer subtypes which have exclusive prognosis [13, 20]. Understanding of the gene expression of breast cancer is notably important in determining the therapeutic interventions; however, the biological behavior of the molecular subtype is largely characterized by the clinico-pathologic features of the tumor [21]. The results of the present study revealed that $62.7 \%$ of primary breast cancers were luminal in nature. In our study, luminal B cancer was more prevalent (69\%) than luminal A (31\%). The results are in concordance with other studies conducted in Italy (34\% luminal A and 36\% luminal B) [22] and Saudi Arabia (3.9\% luminal $\mathrm{A}$ and $16 \%$ luminal B) [23] which found luminal B subtypes more common than luminal A albeit with other studies conducted in various parts of the world including the USA (55\% luminal $\mathrm{A}$ and $17 \%$ luminal B) [24], Tunis (51.5\% luminal A and $16 \%$ luminal B) [25], Japan (71\% luminal $A$ and $8 \%$ luminal B) [26], Egypt (44.3\% luminal A and 24.6\% luminal B) [27], China (65.3\% luminal $A$ and $19 \%$ luminal B) [28], and Algeria (50.6\% luminal A and $19 . \%$ luminal B) [29] which found luminal $\mathrm{A}$ as the most prevalent molecular subtype of breast cancer. The variation in the commonest profile of breast cancer explains the heterogeneity of breast cancer across the world.

Additionally, the clinico-pathologic parameters of luminal A and luminal B breast cancers including age, tumor size, grade, ki67 value, tumor stage, nodal status, and laterality were correlated. The frequency of luminal B subtype was notably higher in a younger age group as compared to luminal $\mathrm{A}$, which was more frequent in older age. Clinically, luminal B cancers are associated with poor outcomes and aggressive behavior even in the presence of concomitant therapy [15]. The highest numbers of patients with luminal B subtype (47\%) were between the age group of 31 to 50 years, making it the most widespread subtype of breast cancer among young-age women. These findings are in congruence with other studies that revealed that the incidence of breast cancer is considerably higher among premenopausal women (below 40 years) in low- and middleincome countries [30]. A similar study conducted in Turkey revealed that approximately half of the patients diagnosed with breast cancer were premenopausal, and $20 \%$ of them were below the age of 40 years [31].

Moreover, $26.5 \%$ of luminal B type cancers were high grade as compared to luminal A cancers. The present study also demonstrated that luminal B cancers were more advancing as more than $50 \%$ of the tumors exhibited nodal metastasis, while in luminal A subtype, only 
Table 1 Co-relation of clinico-pathologic parameters of luminal A and luminal B breast cancers

\begin{tabular}{|c|c|c|c|c|c|}
\hline & & Luminal B & Luminal A & Total & $P$ value \\
\hline Age (years) $\uparrow \pm$ & & $51.12 \pm 12.619$ & $54.63 \pm 12.740$ & & $<0.05$ \\
\hline \multicolumn{6}{|c|}{ Age group $(n=1224)$} \\
\hline & $\leq 30$ & $42(5)$ & $14(3.7)$ & $56(4.6)$ & \multirow[t]{4}{*}{$<0.05$} \\
\hline & $31-50$ & $397(47)$ & 138(36.4) & $535(43.7)$ & \\
\hline & $51-70$ & $355(42)$ & 194(51.2) & $549(44.9)$ & \\
\hline & $>70$ & $51(6)$ & $33(8.7)$ & $84(6.9)$ & \\
\hline \multicolumn{6}{|c|}{ Tumor grade $(n=1224)$} \\
\hline & Grade 1 & $94(11.1)$ & 112(29.6) & 206(16.8) & \multirow[t]{3}{*}{$<0.05$} \\
\hline & Grade 2 & $527(62.4)$ & $235(62)$ & $762(62.3)$ & \\
\hline & Grade 3 & $224(26.5)$ & $32(8.4)$ & $256(20.9)$ & \\
\hline Size of tumor $\uparrow \pm$ & & $35.09 \pm 14.20$ & $35.21 \pm 15.18$ & & 0.985 \\
\hline \multicolumn{6}{|c|}{ Tumor stage $(n=504)$} \\
\hline & $\mathrm{T} 1$ & $41(13.7)$ & $35(17.2)$ & $76(15.1)$ & \multirow[t]{3}{*}{0.535} \\
\hline & $\mathrm{T} 2$ & $216(72)$ & 139(68.1) & $355(70.4)$ & \\
\hline & $\mathrm{T} 3 / \mathrm{T} 4$ & $43(14.3)$ & $30(14.7)$ & $73(14.5)$ & \\
\hline \multicolumn{6}{|c|}{ Nodal status $(n=504)$} \\
\hline & Positive & 162(54) & $89(43.6)$ & 251(49.8) & \multirow[t]{2}{*}{$<0.05$} \\
\hline & Negative & 138(46) & 115(56.4) & $253(50.2)$ & \\
\hline \multicolumn{6}{|l|}{ N stage $(n=504)$} \\
\hline & No & $141(47)$ & $115(56.4)$ & $256(50.8)$ & \multirow[t]{4}{*}{$<0.05$} \\
\hline & N1 & $74(24.7)$ & $50(24.5)$ & 124(24.6) & \\
\hline & N2 & $50(16.7)$ & $16(7.8)$ & $66(13.1)$ & \\
\hline & N3 & $35(11.7)$ & 23(11.3) & $58(11.5)$ & \\
\hline \multicolumn{6}{|c|}{ Laterality $(n=1224)$} \\
\hline & Left & 419(49.6) & 191(50.4) & $610(49.8)$ & \multirow[t]{2}{*}{0.793} \\
\hline & Right & $426(50.4)$ & 188(49.6) & $614(50.2)$ & \\
\hline
\end{tabular}

Chi-square was applied

\pm Mean \pm SD

$\uparrow$ Mann-Whitney test

43\% tumors exhibited nodal metastasis. Apart from tumor grade, axillary involvement is another predominant prognostic factor of breast cancer [27, 32]. In our study, the rate of axillary positivity was more pronounced among younger patients. Alternately, tumor size is used to access prognosis of breast cancer in the absence of nodal involvement [23]. Apropos of tumor characteristics, the luminal B subtype was more aggressive in our study as nodal involvement; tumor grade were significantly high in luminal $\mathrm{B}$. These findings are consistent with another research study which indicated that luminal B cancers are characterized by larger tumor size, extensive nodal involvement, and advanced tumor stage as compared to luminal A subtypes [33].

Lymphovascular invasion is another prognostic factor that determines the risk of recurrence after treatment [34]. Lymphovascular invasion was more frequent in luminal $\mathrm{B}$ cancers, which implies that post-treatment recurrence of disease in luminal $B$ cancers make them more challenging to manage and also reduce the survival rate in these patients. The study also revealed a high frequency of lobular, cribriform, papillary, and mucinous characteristics in luminal A subtype of breast cancers while luminal B cancers were more aggressive in nature on account of advanced tumor grade, nodal involvement, lymphovascular invasion, and higher frequency of micropapillary histology. These findings are consistent with other studies which demonstrated that micropapillary carcinomas are more intrusive in nature due to their propensity to disseminate to axillary lymph nodes and these carcinomas are usually of luminal B type [35, 36]. Moreover, papillary and mucinous phenotypes are more indolent as compared to micropapillary phenotype, thus accounting for the more aggressive nature of luminal B subtypes. 
Table 2 Co-relation of histologic features of luminal A and luminal B breast cancers. Chi-square was applied

\begin{tabular}{|c|c|c|c|c|}
\hline & Luminal B & Luminal A & Total & $P$ value \\
\hline \multicolumn{5}{|c|}{ Lymphocytic infiltration $(n=504)$} \\
\hline Absent & 197(65.7) & $151(74)$ & $348(69)$ & 0.060 \\
\hline Mild to moderate & $85(28.3)$ & $48(23.5)$ & 133(26.4) & \\
\hline Severe & 18(6) & $5(2.5)$ & $23(4.6)$ & \\
\hline \multicolumn{5}{|c|}{ In situ component $(n=503)$} \\
\hline Present & 214(71.6) & $155(76)$ & $369(73.4)$ & 0.272 \\
\hline Absent & $85(28.4)$ & $49(24)$ & 134(26.6) & \\
\hline \multicolumn{5}{|c|}{ Lymphovascular invasion $(n=504)$} \\
\hline Present & $88(29.3)$ & $31(15.2)$ & 119(23.6) & $\leq 0.05$ \\
\hline Absent & $212(70.7)$ & $173(84.8)$ & $385(76.4)$ & \\
\hline \multicolumn{5}{|c|}{ Dermal lymphatic invasion $(n=504)$} \\
\hline Present & $38(12.7)$ & $30(14.7)$ & $68(13.5)$ & 0.511 \\
\hline Absent & $262(87.3)$ & $174(85.3)$ & $436(86.5)$ & \\
\hline \multicolumn{5}{|c|}{ Pagetoid spread $(n=504)$} \\
\hline Present & $5(1.7)$ & $2(1)$ & $7(1.4)$ & 0.707 \\
\hline Absent & 295(98.3) & 202(99) & 497(98.6) & \\
\hline
\end{tabular}

The major limitation of this study is lack of followup/recurrence data; however, detailed histological and immunohistochemical features of a large series of breast cancer presented in our study will be useful in tailoring therapeutic regimens in loco-regional population.

\section{Conclusion}

In conclusion, prognosis and treatment of breast cancer largely depends upon the tumor characteristics including histological features, genetic profile, and clinico-pathologic parameters of the tumor. So, we suggest that each healthcare facility must conduct detailed histological, genetic, and molecular subtyping before tailoring a standard treatment regimen for

Table 3 Distribution of histological types of breast carcinoma among luminal A and luminal B breast carcinoma

\begin{tabular}{llll}
\hline & Luminal B & Luminal A & Total \\
\hline IDC & $747(88.4)$ & $287(75.7)$ & $1034(84.5)$ \\
ILC & $41(4.9)$ & $41(10.8)$ & $82(6.7)$ \\
Cribriform & $3(0.4)$ & $4(1.1)$ & $7(0.6)$ \\
Papillary & $14(1.7)$ & $18(4.7)$ & $32(2.6)$ \\
Mucious & $12(1.4)$ & $22(5.8)$ & $34(2.8)$ \\
Micropapillary & $13(1.5)$ & $0(0)$ & $13(1.1)$ \\
Tubular & $4(0.5)$ & $6(1.6)$ & $10(0.8)$ \\
Metaplastic & $8(0.9)$ & $1(0.3)$ & $9(0.7)$ \\
Mixed & $3(0.4)$ & $0(0)$ & $3(0.2)$ \\
Total & 845 & 379 & 1224 \\
\hline
\end{tabular}

each patient. The results of our study also indicated that luminal $\mathrm{B}$, which is an aggressive type of breast cancer, is more prevalent among younger patients, which demonstrate poor prognosis in younger age as compared to older age.

\section{Acknowledgements}

We gratefully acknowledge all staff members of Pathology, Liaquat National Hospital, Karachi, Pakistan, for their help and cooperation.

\section{Funding \\ No funding was provided.}

\section{Availability of data and materials}

Please contact the author for data requests.

\section{Authors' contributions}

$\mathrm{AAH}, \mathrm{SA}$, and SMK are the main authors of manuscript and have made substantial contributions to the conception and design of study. RM, MIZ, and $\mathrm{NI}$ have been involved in requisition of data. MN, MS, NF, MME, and AK have been involved in analysis of the data and gave final approval and revised of the manuscript. All authors read and approved the final manuscript.

\section{Ethics approval and consent to participate}

The Ethics committee of Liaquat National Hospital, Karachi, Pakistan, approved the study. Written informed consent was obtained from the patients for their participation.

\section{Consent for publication}

The written consent is available for review by the Editor-in-Chief of this journal.

\section{Competing interests}

The authors declare that they have no competing interests.

\section{Publisher's Note}

Springer Nature remains neutral with regard to jurisdictional claims in published maps and institutional affiliations.

\section{Author details}

${ }^{1}$ Liaquat National Hospital and Medical College, Karachi, Pakistan. ${ }^{2}$ Shaheed Zulfiqar Ali Bhutto Institute of Science and Technology, Karachi, Pakistan. ${ }^{3}$ Aga Khan University, Karachi, Pakistan. ${ }^{4}$ Department of Pathology, Liaquat National Hospital and Medical College, Karachi, Pakistan. ${ }^{5}$ Department of Surgery, Brown University, Providence, RI, USA. ${ }^{6}$ Department of Medicine, Kandahar University, Kandahar, Afghanistan.

Received: 15 August 2017 Accepted: 18 December 2017

Published online: 02 January 2018

\section{References}

1. Fan C, Oh DS, Wessels $L$, et al. Concordance among gene-expression-based predictors for breast cancer. N Engl J Med. 2006;355:560.

2. Hu Z, Fan C, Oh DS, et al. The molecular portraits of breast tumors are conserved across microarray platforms. BMC Genomics. 2006;7:96.

3. Voduc KD, Cheang MC, Tyldesley S, et al. Breast cancer subtypes and the risk of local and regional relapse. J Clin Oncol. 2010;28:1684.

4. Goldhirsch A, Winer EP, Coates AS, Gelber RD, Piccart-Gebhart M, Thürlimann B, Senn HJ. Panel members: personalizing the treatment of women with early breast cancer: highlights of the St Gallen International Expert Consensus on the Primary Therapy of Early Breast Cancer 2013. Ann Oncol. 2013;24:2206-23.

5. Petric M, Martinez S, Acevedo F, Oddo D, Artigas R, Camus M, Sanchez C. Correlation between Ki67 and histological grade in breast cancer patients treated with preoperative chemotherapy. Asian Pac J Cancer Prev. 2014; 15(23):10277-80. 
6. Cheang MCU, Chia SK, Voduc D, et al. Ki67 index, HER2 status, and prognosis of patients with luminal B breast cancer. J Natl Cancer Inst. 2009;101:736-50.

7. Prat A, Cheang MC, Martin M, et al. Prognostic significance of progesterone receptor-positive tumor cells within immunohistochemically defined luminal A breast cancer. J Clin Oncol. 2013:31:203-9.

8. Hashmi AA, Edhi MM, Naqvi H, Khurshid A, Faridi N. Molecular subtypes of breast cancer in South Asian population by immunohistochemical profile and Her2neu gene amplification by FISH technique: association with other clinicopathologic parameters. Breast J. 2014;20(6):578-85.

9. Hashmi AA, Edhi MM, Naqvi H, Faridi N, Khurshid A, Khan M. Clinicopathologic features of triple negative breast cancers: an experience from Pakistan. Diagn Pathol. 2014;9:43.

10. Haroon S, Hashmi AA, Khurshid A, Kanpurwala MA, Mujtuba S, Malik B, Faridi N. Ki67 index in breast cancer: correlation with other prognostic markers and potential in Pakistani patients. Asian Pac J Cancer Prev. 2013;14(7):4353-8

11. Salgado R, Denkert C, Demaria S, Sirtaine N, Klauschen F, Pruneri G et. al. The evaluation of tumor-infiltrating lymphocytes (TILS) in breast cancer: recommendations by an International TILs Working Group 2014. Ann Oncol 2015:26(2):259-271.

12. Rosen PP. Tumor emboli in intramammary lymphatics in breast carcinoma: pathologic criteria for diagnosis and clinical significance. Pathol Annu. 1983: 18 Pt 2:215-32.

13. Collins LC, Botero ML, Schnitt SJ. Bimodal frequency distribution of estrogen receptor immunohistochemical staining results in breast cancer. Am J Clin Pathol. 2005;123:16-20.

14. McCarty KS Jr, Miller LS, Cox EB, et al. Estrogen receptor analyses: correlation of biochemical and immunohistochemical methods using monoclonal antireceptor antibodies. Arch Pathol Lab Med. 1985;109(8):716-21.

15. Wolff AC, Hammond ME, Schwartz JN, et al. American Society of Clinical Oncology/College of American Pathologists guideline recommendations for human epidermal growth factor receptor 2 testing in breast cancer. Arch Pathol Lab Med. 2007;131(1):18-43.

16. Wolff AC, Hammond ME, Hicks DG, et al. Recommendations for human epidermal growth factor receptor 2 testing in breast cancer: American Society of Clinical Oncology_College of American Pathologists (ASCO/CAP) Clinical Practice Guideline Update (2013). Arch Pathol Lab Med. In press

17. Agarwal G, Pradeep PV, Aggarwal V, Yip CH, Cheung PS. Spectrum of breast cancer in Asian women. World J Surg. 2007:31(5):1031-40.

18. Hashmi AA, Faridi N, Khurshid A, Naqvi H, Malik B, Malik FR, Fida Z, Mujtuba S. Accuracy of frozen section analysis of sentinel lymph nodes for the detection of Asian breast cancer micrometastasis - experience from Pakistan. Asian Pac J Cancer Prev. 2013;14(4):2657-62.

19. Fan $C$, Oh DS, Wessels $L$, Weigelt $B$, Nuyten DS, Nobel AB, Van't Veer $L$, Perou CM. Concordance among gene-expression-based predictors for breast cancer. N Engl J Med. 2006;355(6):560-9.

20. Cheang MC, Chia SK, Voduc D, Gao D, Leung S, Snider J, Watson M, Davies S, Bernard PS, Parker JS, Perou CM. Ki67 index, HER2 status, and prognosis of patients with luminal B breast cancer. JNCl: Journal of the National Cancer Institute. 2009;101(10):736-50.

21. Özmen V. Breast cancer in Turkey: clinical and histopathological characteristics (analysis of 13.240 patients). The Journal of Breast Health. 2014 Apr;10(2):98.

22. Caldarella A, Buzzoni C, Crocetti E, Bianchi S, Vezzosi V, Apicella P, Biancalani M, Giannini A, Urso C, Zolfanelli F, Paci E. Invasive breast cancer: a significant correlation between histological types and molecular subgroups. J Cancer Res Clin Oncol. 2013;139(4):617-23.

23. Al Tamimi DM, Shawarby MA, Ahmed A, Hassan AK, AlOdaini AA. Protein expression profile and prevalence pattern of the molecular classes of breast cancer-a Saudi population based study. BMC Cancer. 2010;10(1):223.

24. Bhargava R, Striebel J, Beriwal S, Flickinger JC, Onisko A, Ahrendt G, Dabbs DJ. Prevalence, morphologic features and proliferation indices of breast carcinoma molecular classes using immunohistochemical surrogate markers. Int J Clin Exp Pathol. 2009;2(5):444.

25. Abdelkrim SB, Trabelsi A, Missaoui N, Beizig N, Bdioui A, Anjorin A, Jomaa W, Mokni M. Distribution of molecular breast cancer subtypes among Tunisian women and correlation with histopathological parameters: a study of 194 patients. Pathology-Research and Practice. 2010;206(11):772-5.

26. Shibuta K, Ueo H, Furusawa H, Komaki K, Rai Y, Sagara Y, Kamada Y, Tamaki $\mathrm{N}$. The relevance of intrinsic subtype to clinicopathological features and prognosis in 4,266 Japanese women with breast cancer. Breast Cancer. 2011:18(4):292-8.

27. Salhia B, Tapia C, Ishak EA, Gaber S, Berghuis B, Hussain KH, DuQuette RA, Resau J, Carpten J. Molecular subtype analysis determines the association of advanced breast cancer in Egypt with favorable biology. BMC Womens Health. 2011;11(1):44

28. Zhu X, Ying J, Wang F, Wang J, Yang H. Estrogen receptor, progesterone receptor, and human epidermal growth factor receptor 2 status in invasive breast cancer: a 3,198 cases study at National Cancer Center, China. Breast Cancer Res Treat. 2014;147(3):551-5

29. Cherbal F, Gaceb H, Mehemmai C, Saiah I, Bakour R, Rouis AO, Boualga K, Benbrahim W, Mahfouf H. Distribution of molecular breast cancer subtypes among Algerian women and correlation with clinical and tumor characteristics: a population-based study. Breast disease. 2015;35(2):95-102.

30. Brinton LA, Sherman ME, Carreon JD, Anderson WF. Recent trends in breast cancer among younger women in the United States. JNCl: Journal of the National Cancer Institute. 2008;100(22):1643-8.

31. Ordu C, McGuire K, Alco G, Pilanci KN, Koksal UI, Elbüken F, Erdogan Z, Agacayak F, llgun S, Sarsenov D, Öztürk A. The prognostic impact of molecular subtypes and very young age on breast conserving surgery in early stage breast cancer. Cureus. 2016;8(6):e633.

32. Weigel MT, Dowsett M. Current and emerging biomarkers in breast cancer: prognosis and prediction. Endocr Relat Cancer. 2010 Dec 1;17(4):R245-62.

33. Cancello G, Maisonneuve P, Rotmensz N, Viale G, Mastropasqua MG, Pruner G, Montagna E, lorfida M, Mazza M, Balduzzi A, Veronesi P. Progesterone receptor loss identifies luminal $B$ breast cancer subgroups at higher risk of relapse. Ann Oncol. 2012;24(3):661-8.

34. Rosen PR, Groshen S, Saigo PE, Kinne DW, Hellman S. A long-term follow-up study of survival in stage I (T1NOM0) and stage II (T1N1M0) breast carcinoma. J Clin Oncol. 1989;7(3):355-66.

35. Horlings HM, Weigelt B, Anderson EM, Lambros MB, Mackay A, Natrajan R, Ng CK, Geyer FC, van de Vijver MJ, Reis-Filho JS. Genomic profiling of histological special types of breast cancer. Breast Cancer Res Treat. 2013; 142(2):257-69.

36. Weigelt B, Horlings HM, Kreike B, Hayes MM, Hauptmann M, Wessels LF, De Jong D, Van de Vijver MJ, Van't Veer $L$, Peterse JL. Refinement of breast cancer classification by molecular characterization of histological special types. J Pathol. 2008;216(2):141-50.

\section{Submit your next manuscript to BioMed Central and we will help you at every step:}

- We accept pre-submission inquiries

- Our selector tool helps you to find the most relevant journal

- We provide round the clock customer support

- Convenient online submission

- Thorough peer review

- Inclusion in PubMed and all major indexing services

- Maximum visibility for your research

Submit your manuscript at www.biomedcentral.com/submit
) Biomed Central 